\title{
Extraction and biological evaluation of Mycobacterium kansasii extracellular vesicles as a vaccine candidate against mycobacterial pulmonary infections
}

\author{
Hoseini Tavassol $\mathbf{Z}^{1}$, Vaziri $\mathrm{F}^{2,3}$, Siadat $\mathrm{SD}^{2,3 *}$
}

\author{
${ }^{1}$ Department of Biology, Science and Research Branch, Islamic Azad University, Tehran, I. R. Iran. \\ ${ }^{2}$ Department of Mycobacteriology and Pulmonary Research, Pasteur Institute of Iran, Tehran, I. R. Iran. \\ ${ }^{3}$ Microbiology Research Center, Pasteur Institute of Iran, Tehran, I. R. Iran.
}

\begin{abstract}
Introduction: Extracellular vesicles (EVs) are bacterial products with diverse biological roles. Like many microorganisms, Mycobacterium kansasii as a nontuberculous mycobacteria (NTM), can naturally release EVs. The aim of the present study was the extraction and biological evaluation of $M$. kansasii as a vaccine candidate against mycobacterial pulmonary infections. Methods: After bacterial culture of the standard species of $M$. kansasii, the EVs extraction was done by density gradient ultra-centrifugation method and biological evaluations of EVs were performed by SDS-PAGE and electron microscopy. Endotoxin safety of the EVs was evaluated by LAL test. Results: SDS-PAGE result showed more than 5 prominent protein bands $(60-180 \mathrm{kDa})$. The intactness of the vesicles was verified by electron microscopy through which the spherical configuration of EVs with diameter of 200-300 nm could be observed. The amount of lipopolysaccharide (LPS) contamination existing in EVs was in the specified application range of biological products. Conclusion: EVs were prepared with acceptable quality composition with intact conformational structure throughout the extraction procedure. The extracted EVs had the initial requirements as an immunogenic molecule, such as safety, stability, inexpensiveness and antigens possession which based on the similarities between M. kansasii and M. tuberculosis, make them a suitable candidate for future prophylactics, therapeutic, detection and adjuvants studies against mycobacterial pulmonary infections.
\end{abstract}

KEYWORDS: Extracellular vesicles (EVs), Mycobacterium kansasii, adjuvant.

\section{INTRODUCTION}

Previous studies have shown that bacteria release extracellular vesicles (EVs) naturally, also known as, outer membrane vesicles and membrane vesicles, under a variety of growth situations [1]. EVs are accounted for distinctive features such as, interaction of the microorganism with the environment, as well as the microbe's physiology and pathogenesis, enzymes and toxins delivery, communication signals, presentation of recognized antigens to innate and adaptive immune system, defense against other microorganisms, resistance to host immune system, nutrition and survival, transfer of certain genes and biofilm formation [2-4]. EVs diverse biological roles, probably make them one of the bacterial evolution factors. Furthermore, EVs can be considered as a non-classical secretory system [5]. Considering the EVs capability to concentrate toxins and immune-modulatory molecules, the production of EVs is related to bacterial virulence. In this manner, the production of mycobacterial vesicles as a delivery mechanism of immunologically active molecules can lead to

*Corresponding Author: Seyed Davar Siadat. Bacteriology Ph.D, Department of Mycobacteriology and Pulmonary Research, Pasteur Institute of Iran, Tehran, I. R. Iran.

Email: d.siadat@gmail.com

Tel/Fax: (+98) 2166953311-20 mycobacterial virulence which can be exploited to diagnose the pathogenic mycobacteria and the development of vaccines against them [6].

Like many other microorganisms, Mycobacterium (M.) kansasii naturally releases EVs in a variety of growth media. $M$. kansasii is the second most common cause of nontuberculous mycobacteria (NTM) infections [7, 8]. Although more than 150 different species of NTM have so far been identified, $M$. avium complex (MAC), M kansasii and M. abscessus are the causative agents of most pulmonary infections [9]. Buhler and Pollak identified M. kansasii for the first time in 1953 and named it "yellow bacillus". M. kansasii antigenic and clinical characteristics make it the most similar Mycobacterium to $M$. tuberculosis [10].

Due to the prevalence of NTM infections, studies to develop awareness about these microorganisms are important in order to prevent the disease from spreading among the high-risk individuals such as HIV patients. Mycobacterial extracts have been widely used in vaccines and adjuvants, such as Freund's complete adjuvant. Mycobacterial cell wall contains a variety of antigens and the immune stimulating biomolecules, such as peptidoglycan, arabinogalactan, mycolic acids, phosphatidylinositol mannosides, tiocerol, lipomanann, and lipoarabinomann which can activate the dendritic cells. All 
these compounds are effective molecular factors in the process of tuberculosis in mice and can induce immune responses against the infections. EVs derived from non-pathogenic mycobacteria such as $M$. bovis and $M$. smegmatis show high level of consistency with $M$. tuberculosis antigens. These remarkable property makes the EVs a suitable candidate for the next generation of vaccines or adjuvants [11]. Proteomic analyses of $M$. tuberculosis EVs have revealed approximately 50 proteins related to the virulence of the bacterium. These observations show that EVs can be used as an alternative to the active immunological $M$. tuberculosis virulence factors and could be used as a biomarker. Furthermore, it could be potentially used as a vaccine with no need to adjuvants [1]. The aim of the present study was the extraction and biological evaluation of $M$. kansasii EVs as a vaccine candidate.

\section{MATERIALS and METHODS}

\section{Bacteria strain and culture condition}

Standard strain of M. kansasii (CRBIP 7.42) was obtained from the standard bacterial collection of Mycobacteriology and Pulmonary Research Department, Pasteur Institute of Iran. The bacterial culture was initially grown in Lowenstein-Jensen medium (Pasteur Institute of Iran, Tehran, Iran) in order to activate the standard strain. After early preparation, the bacteria adjusted to $1 \mathrm{McFarland}$ turbidity were cultured in $150 \mathrm{ml}$ of Middlebrook $7 \mathrm{H} 9$ broth medium (Sigma, USA) at $37 \pm 1^{\circ} \mathrm{C}$ for 4 weeks. The biomass was then harvested and the wet weight was evaluated.

\section{EVs extraction}

The EVs extraction and purification were performed by density gradient ultra-centrifugation method (Claassen's method), using 3 extraction solutions. Firstly, $0.1 \mathrm{M}$ Tris- $\mathrm{HCl}$ (Sigma, USA), $\mathrm{pH} 8.6 \pm 0.1$ and $10 \mathrm{mM}$ EDTA (Sigma, UK) solution, secondly, 0.1 M Tris-HCl, pH 8.6 0.1 and $10 \mathrm{mM}$ EDTA and $100 \%$ (w/v) deoxycholate (Merck, Darmstadt, Germany) solution and thirdly, $0.1 \mathrm{M}$ Tris-HCl, $\mathrm{pH} 8.6 \pm 0.1$ and $10 \mathrm{mM}$ EDTA and $0.5 \% \mathrm{w} / \mathrm{v}$ deoxycholate solution $[12,13]$.

After deactivation of the bacterium for $2 \mathrm{~h}$ at $80^{\circ} \mathrm{C}$, the cells were harvested by centrifugation at $1000 \mathrm{x} \mathrm{g}$ twice for $30 \mathrm{~min}$. Then the supernatant was suspended in normal saline for 30 min and centrifuged at $1000 \mathrm{x} \mathrm{g}$ for $30 \mathrm{~min}$. The bacterial biomass was suspended in the first solution at 7.5 times of the wet weight for $30 \mathrm{~min}$ and then it was severely re-suspended by adding 1/20th volume of the second solution for $10 \mathrm{~min}$. Finally, the suspension was centrifuged at $16000 \mathrm{x}$ g at $4^{\circ} \mathrm{C}$ for $90 \mathrm{~min}$. The obtained supernatant containing the EVs was concentrated by ultracentrifugation at $60000 \mathrm{x} \mathrm{g}$ at $4^{\circ} \mathrm{C}$ for 120 min and at $48000 \mathrm{x} \mathrm{g}$ at $4^{\circ} \mathrm{C}$ for $180 \mathrm{~min}$. The EVs pellet was re-suspended in the third solution and was centrifuged at 60000 $\mathrm{x} \mathrm{g}$ at $4^{\circ} \mathrm{C}$ for $120 \mathrm{~min}$. The final pellet of EVs was resuspended in $3 \%$ sucrose solution.

\section{Physiochemical analysis}

\section{Protein assay}

After the extraction, the protein concentration of EVs was measured by Nano-drop technique and Bradford protein assay. The Nano-drop protein concentration method uses absorbance at $280 \mathrm{~nm} \quad$ (NanoDrop $^{\mathrm{TM}} 1000$ Spectrophotometer, ThermoScientific Co. USA) [13]. Determination of EVs proteins by Bradford Coomassie brilliant blue assay was confirmed by measuring absorbance at $590 \mathrm{~nm}$ [14].

\section{SDS-PAGE}

The protein ingredients of EVs was analyzed by SDS-PAGE in
12\% gel (using a Mini-PROTEAN@ Tetra Cell - Bio-Rad) and the gel was stained with Coomassie Brilliant Blue (CBB).[13]

Electron microscopy

The intactness of the vesicles was checked by Scanning Electron Microscopy (SEM). For this purpose, field emission scanning electron microscope was used (FE-SEM; HITACHI S4160 model with $5 \mathrm{~nm}$ image resolution, 20-30000 $\mathrm{x}$ magnification and maximum accelerating voltage of $30 \mathrm{kV}$ ). First, a small amount of suspension sample was coated with gold and then was observed with the electron microscope.

The Limulus Amebocyte Lysate (LAL) assay

Endotoxin safety was evaluated by LAL test (Pierce ${ }^{\circledR}$ LAL Chromogenic Endotoxin Quantitation Kit, Thermo Fisher Scientific Co. USA; 88282), according to the manufacturer's instructions [13].

\section{RESULTS}

\section{Physiochemical analysis \\ Protein assay}

After extraction of EVs from $1.52 \mathrm{~g}$ harvested bacterial biomass, the total protein output was determined by Nano-drop technique to be $1.4 \mathrm{mg} / \mathrm{ml}$. This result was also confirmed by Bradford protein assay.

\section{SDS-PAGE}

According to the SDS-PAGE result, we had more than 5 prominent protein bands with $\mathrm{Mw}$ between 60-180 kDa (Fig. $1)$.

\section{Electron microscopy}

The intactness of the vesicles was evaluated by SEM, and the EVs configuration revealed spherical bodies with a diameter of 200-300 nm (Fig. 2).

\section{LAL assay}

The amount of lipopolysaccharide (LPS) contamination existing in EVs was under $300 \mathrm{IU}$, which was within the specified application range of the biological products.

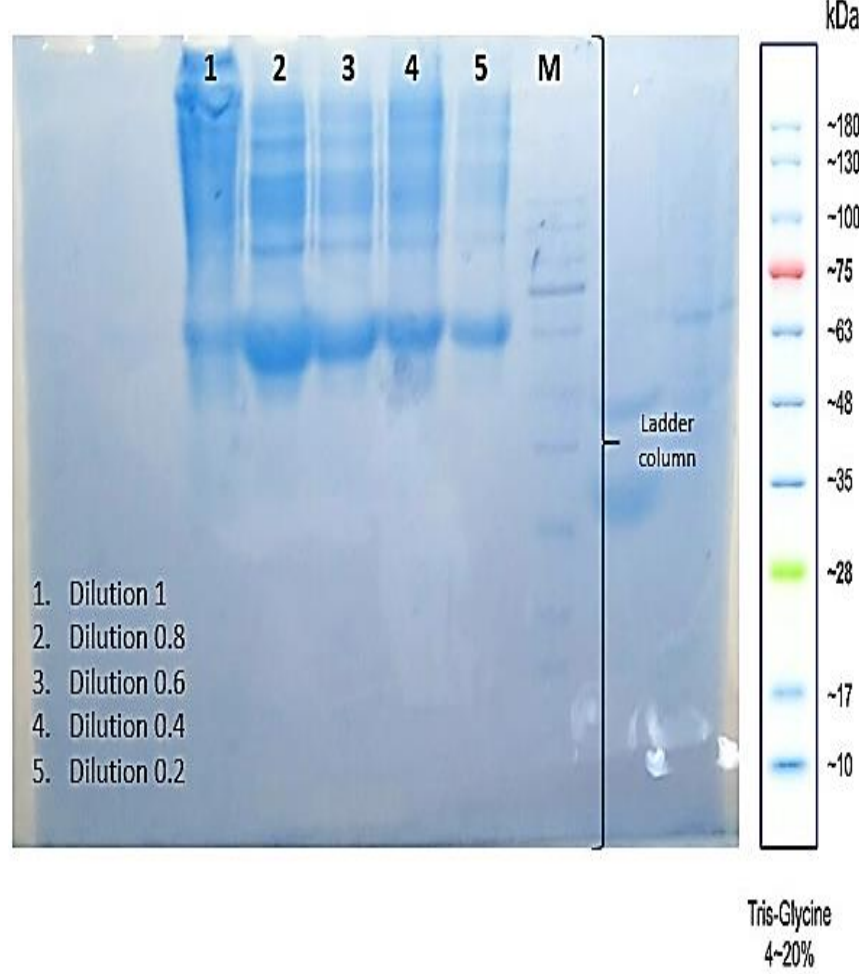

Fig. 1. SDS- PAGE of M. kansasii EVs. 


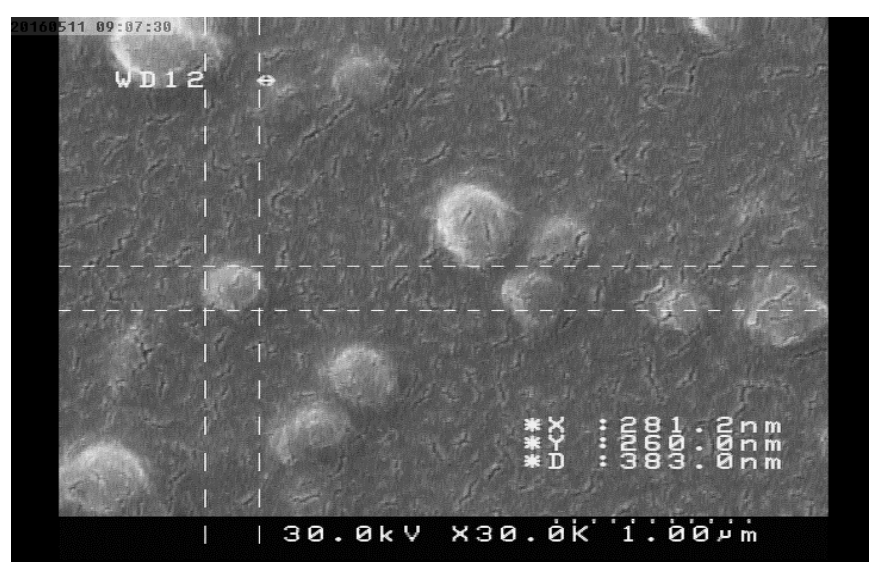

Fig. 2. Electron micrograph of M. kansasii EVs.

\section{DISCUSSION}

Previous studies have shown that important species of mycobacteria produce EVs [6]. Since the EVs play diverse roles in signaling and pathogenicity of bacteria, we believe that the current study of EVs of $M$. kansasii may open new perspectives for further scientific advances in detection, prevention and treatment of infections caused by EV-producing bacteria. Nowadays, with prevalence of the immunocompromising diseases as well as increased incidences of antibiotic resistance in bacteria, the possibility of infections by less known microorganisms such as NTMs has increased. In this regard, recent studies conducted at Mycobacteriology and Pulmonary Research department of Pasteur Institute of Iran has indicated that industrialization and life style changes have led to increased prevalence of $M$. kansasii compared to $M$. tuberculosis infections [15].

Claassen's method is a well-known process for extraction of outer membrane vesicles from Gram-negative bacteria [9]. In this study, we modified this method for extraction of EVs from M. kansasii as a Gram-positive bacterium with its characteristic cell wall. EVs were prepared with high quality composition and their conformational structure remained intact throughout the extraction procedure. The extracted EVs had the initial requirements as an immunogenic molecule, such as safety, stability, inexpensiveness and antigens possession.

Previous proteomic analyses have shown that only EVs from pathogenic strains of mycobacteria are TLR2 lipoprotein agonists. The interaction of the membrane vesicles with macrophages isolated from mice are shown to stimulate the release of cytokines and chemokines in a TLR2-dependent manner while the injection of the EVs into the lung of the mice leads to inflammatory response [6]. Therefore, it can be envisaged that extracted M. kansasii EVs could possibly be used as an immunosuppressive drug to prevent sever immune reactions, especially in lungs.

Reviews on bacterial vesicles and their vaccine applications demonstrate that these vesicles have being used increasingly for development of vaccines. Mycobacterial cell wall contains a variety of antigens and the immune stimulating molecules, such as peptidoglycan, arabinogalactan, mycolic acids, proteins, phosphatidylinositol mannosides, tiocerol, lipomanann, and lipoarabinomann activating dendritic cells. EVs derived from non-pathogenic mycobacteria such as $M$. bovis and $M$. smegmatis exhibit high level of consistency with $M$. tuberculosis antigens [11]. Considering the similarities between M. kansasii and M. tuberculosis, the extracted M. kansasii EVs with its biological, antigenic and clinical characteristics can be considered as a vaccine candidate against mycobacterial pulmonary infections.

Moreover, EVs are recently considered as new generation of adjuvants. Salmani and colleagues have pointed to the necessity of developing more efficient vaccines with less side effects with new and improved adjuvant and have proposed the use of EVs as an adjuvant [16]. Mycobacterial extractions including Freund's complete adjuvant have been widely used in producing vaccines. Upon further verifications and experiments, biological compounds like M. kansasii EVs, can also be suggested as a new adjuvant which may replace chemical compounds such as alum in future vaccine designs.

\section{ACKNOWLEDGEMENT}

The authors gratefully acknowledge Pasteur Institute of Iran for supporting this study.

\section{CONFLICT OF INTEREST}

The authors declare that they have no conflict of interest.

\section{REFERENCES}

1. Lee J, Kim SH, Choi DS, Lee JS, Kim DK, Go G et al. Proteomic analysis of extracellular vesicles derived from Mycobacterium tuberculosis. Proteomics. 2015;15(19):3331-7.

2. Deatherage BL, Cookson BT. Membrane vesicle release in bacteria, eukaryotes, and archaea: a conserved yet underappreciated aspect of microbial life. Infection and Immunity. 2012;80(6):1948-57. doi:10.1128/iai.06014-11.

3. Kuehn MJ, Kesty NC. Bacterial outer membrane vesicles and the hostpathogen interaction. Genes \& development. 2005;19(22):2645-55. doi:10.1101/gad.1299905.

4. Kulp A, Kuehn MJ. Biological functions and biogenesis of secreted bacterial outer membrane vesicles. Annual review of microbiology. 2010;64:163-84. doi:10.1146/annurev.micro.091208.073413.

5. Lee EY, Choi DY, Kim DK, Kim JW, Park JO, Kim S et al. Gram $\square$ positive bacteria produce membrane vesicles: Proteomics $\square$ based characterization of Staphylococcus aureus $\square$ derived membrane vesicles Proteomics. 2009;9(24):5425-36.

6. Prados-Rosales R, Baena A, Martinez LR, Luque-Garcia J, Kalscheuer R, Veeraraghavan $U$ et al. Mycobacteria release active membrane vesicles that modulate immune responses in a TLR2-dependent manner in mice. The Journal of clinical investigation. 2011;121(4):1471-83. doi:10.1172/jci44261.

7. Moon SM, Park HY, Jeon K, Kim SY, Chung MJ, Huh HJ et al. Clinical Significance of Mycobacterium kansasii Isolates from Respiratory Specimens. PLoS ONE. 2015;10(10). doi:10.1371/journal.pone.0139621.

8. Griffith DE, Aksamit T, Brown-Elliott BA, Catanzaro A, Daley C, Gordin $F$ et al. An Official ATS/IDSA Statement: Diagnosis, Treatment, and Prevention of Nontuberculous Mycobacterial Diseases. American Journal of Respiratory and Critical Care Medicine. 2007;175(4):367-416. doi:10.1164/rccm.200604-571ST.

9. Johnson MM, Odell JA. Nontuberculous mycobacterial pulmonary infections. Journal of Thoracic Disease. 2014;6(3):210-20. doi:10.3978/j.issn.2072-1439.2013.12.24

10. Canueto-Quintero J, Caballero-Granado FJ, Herrero-Romero M, Dominguez-Castellano A, Martin-Rico P, Verdu EV et al. Epidemiological, clinical, and prognostic differences between the diseases caused by Mycobacterium kansasii and Mycobacterium tuberculosis in patients infected with human immunodeficiency virus: a multicenter study. Clinical infectious diseases : an official publication of the Infectious Diseases Society of America. 2003;37(4):584-90. doi:10.1086/376987.

11. Acevedo R, Fernández S, Zayas C, Acosta A, Sarmiento ME, Ferro VA et al. Bacterial Outer Membrane Vesicles and Vaccine Applications. Front Immunol. 2014;5. doi:10.3389/fimmu.2014.00121.

12. Claassen I, Meylis J, van der Ley P, Peeters C, Brons H, Robert J et al. Production, characterization and control of a Neisseria meningitidis 
hexavalent class 1 outer membrane protein containing vesicle vaccine. Vaccine. 1996;14(10):1001-8.

13. Delbaz SA, Siadat SD, Aghasadeghi MR, Piryaie M, Peerayeh SN, Mousavi SF et al. Biological and Immunological Evaluation of Neisseria meningitidis Serogroup A Outer Membrane Vesicle as Vaccine Candidates. Jundishapur Journal of Microbiology. 2013;6(4)

14. Bradford MM. A rapid and sensitive method for the quantitation of microgram quantities of protein utilizing the principle of protein-dye binding. Analytical biochemistry. 1976;72(1-2):248-54.
15. Nour-Neamatollahie A, Ebrahimzadeh N, Siadat SD, Vaziri F, Eslami M, Akhavan Sepahi A et al. Distribution of non-tuberculosis mycobacteria strains from suspected tuberculosis patients by heat shock protein 65 PCRRFLP. Saudi Journal of Biological Sciences. 2016 doi:http://dx.doi.org/10.1016/j.sjbs.2016.02.001.

16. Salmani AS, Siadat SD, Norouzian D, Mobarakeh JI, Kheirandish M, Zangeneh $M$ et al. Outer membrane vesicle ofNeisseria meningitidis serogroup B as an adjuvant to induce specific antibody response against the lipopolysaccharide ofBrucella abortus S99. Annals of microbiology. 2009;59(1):145-9. 